\title{
A simple mathematical model for anomalous diffusion via Fisher's information theory
}

\author{
Marcelo R. Ubriaco* \\ Laboratory of Theoretical Physics \\ Department of Physics \\ University of Puerto Rico \\ Río Piedras Campus \\ San Juan \\ PR 00931, USA
}

\begin{abstract}
Starting with the relative entropy based on a previously proposed entropy function $S_{q}[p]=\int d x p(x)(-\ln p(x))^{q}$, we find the corresponding Fisher's information measure. After function redefinition we then maximize the Fisher information measure with respect to the new function and obtain a differential operator that reduces to a space coordinate second derivative in the $q \rightarrow 1$ limit. We then propose a simple differential equation for anomalous diffusion and show that its solutions are a generalization of the functions in the Barenblatt-Pattle solution. We find that the mean squared displacement, up to a $q$-dependent constant, has a time dependence according to $\left\langle x^{2}>\sim K^{1 / q} t^{1 / q}\right.$, where the parameter $q$ takes values $q=\frac{2 n-1}{2 n+1}$ (superdiffusion) and $q=\frac{2 n+1}{2 n-1}$ (subdiffusion), $\forall n \geq 1$.
\end{abstract}

PACS number(s): 89.70.Cf, 05.20.-y

Keywords: entropy, Fisher information, anomalous diffusion

\section{Introduction}

During the past two decades the Fisher information measure [1 has become, due mainly to the work of Frieden and coworkers, to play a fundamental role from where the well known covariant field equations of physics can be extracted [2- [4]. Although the Fisher information measure is obtained from the relative entropy, it plays a different role than the Shannon's information measure. As physics

*Electronic address:ubriaco@ltp.upr.clu.edu 
is concerned, in statistical mechanics maximization of Shannon's entropy under some constraints gives us the probability distribution function and therefore the partition function for a particular system, while in the Fisher information measure after defining a new function $\phi(p)$ in terms of the probability amplitudes $p(x)$, one obtains a 'kinetic' term in terms of $\phi$ leading to the interpretation of the Fisher information as a kind of lagrangian. In particular, in Refs. 22-4] it has been shown that the familiar relativistic wave equations are obtained maximizing the expressions $I-J$, where $I$ is the Fisher measure in term of the new functions and $J$ is obtained from the Fourier transform of $I$ and use of the energy constraint. Curiously, this approach works very well for the case of covariant formulations. For the nonconvariant case, as it is the case of the Schrödinger and the diffusion equations, the Fisher measure defines the space coordinates differential operator and thus the time part has to be introduced by using dimensional analysis.

This paper is organized as follows. In Section (2) we review the relation of the Fisher measure and the Kullback entropy, and briefly explain how the definition of the new function $\phi$ as a function of $p(x)$ leads to the interpretation of $I$ as a lagrangian. In Section (3) we introduce the relative entropy for our entropy function and we obtain, following the method in Ref [5]), the corresponding Fisher measure, and then after function redefinition we find coordinate differential operator. In this section we also obtain the eigenfunctions of this differential operator. In Section (4) we show that this formalism the corresponding leads to an alternative differential equation for anomalous diffusion. In Section (5) we discuss our results.

\section{Fisher information measure as a lagrangian}

The Fisher information measure is defined through the integral

$$
I=\int d x\left(\frac{d p(x)}{d x}\right)^{2} p^{-1}(x),
$$

where $p(x)$ denotes the probability distribution. In Ref. 5 it was shown that $I$ can be obtained by considering a small global shift $\Delta$ of $p(x)$ in the Kullback information as follows 1

$$
I(\Delta)=\int d x p(x) \ln [p(x) / p(x+\Delta)],
$$

and expanding in series in powers of the shift $\Delta$, leading to the equation

$$
I(\Delta)=-\int d x\left[\frac{d p}{d x} \Delta-\left(\frac{\left(\frac{d p}{d x}\right)^{2}}{p}-\frac{d^{2} p}{d x^{2}}\right) \frac{\Delta^{2}}{2}+\ldots\right],
$$

where one can recognize the Fisher measure in the quadratic term. In 1943, Fisher introduced [6] in Equation (11) a new function $\phi(x)$, called probability

\footnotetext{
${ }^{1}$ An alternative derivation is given in Ref. [2]
} 
amplitudes, in terms of $p(x)$ through the relation $p(x)=\phi^{2}(x)$ leading to the simpler equation 2

$$
I=4 \int d x \frac{d \phi(x)}{d x} \frac{d \phi(x)}{d x},
$$

which is the starting point of Frieden's approach [3] to derive the equations that describe the behavior of physical systems. Introducing space-time coordinates, allowing two real components $\phi_{1}$ and $\phi_{2}$, and after defining $\Phi=\phi_{1}+i \phi_{2}$ Equation (4) was promoted to a covariant expression [4]

$$
I=4 \int d^{4} x \partial_{\nu} \Phi^{*} \partial^{\nu} \Phi
$$

such that performing the Fourier transform of $I$ give us the energy constraint and thus the mass term $m^{2} \Phi^{*} \Phi$, leading to the well known Klein-Gordon equation.

\section{Fisher information based on fractional calcu- lus inspired entropy}

In a previous article [7, based on the definition of the Riemann-Liouville fractional derivative, we proposed a new entropy function defined as

$$
S_{q}[p]=\lim _{t \rightarrow-1} \frac{d}{d t}\left({ }_{-\infty} D_{t}^{q-1} \sum_{i} e^{-t \ln p_{i}}\right),
$$

where $0<q<1$, and the fractional derivative is given by

$$
{ }_{a} D_{t}^{q}=\left(\frac{d}{d t}\right)^{n}\left({ }_{a} D_{t}^{q-n} f(t)\right)
$$

with $n \in N$ and $n>q$. The equation

$$
{ }_{a} D_{t}^{q-n} f(t)=\frac{1}{\Gamma(n-q)} \int_{a}^{t} \frac{f\left(t^{\prime}\right)}{\left(t-t^{\prime}\right)^{(1+q-n)}} d t^{\prime} .
$$

defines the fractional integral operator. Equation (6) leads to the entropy function

$$
S_{q}[p]=\sum_{i} p_{i}\left(-\ln p_{i}\right)^{q}
$$

which is a concave function and satisfies the Lesche and thermodynamic stability criteria. As explained in [7], the motivation to propose Equation (6) as a definition of a new entropy function is based on the observation that the Shannon entropy and Tsallis entropy can be defined according to [8]

$$
\begin{aligned}
S & =\lim _{t \rightarrow-1} \frac{d}{d t} \sum_{i} p_{i}^{-t} \\
S_{q} & =\lim _{t \rightarrow-1} D_{q}^{t} \sum_{i} p_{i}^{-t},
\end{aligned}
$$

\footnotetext{
${ }^{2}$ Fisher used the symbol $q(x)$ instead of $\phi(x)$, which we will not use to avoid confusion with the parameter $q$.
} 
where the operator

$$
D_{q}^{t}=t^{-1} \frac{1-q^{t d / d t}}{1-q} .
$$

is called the Jackson q-derivative [9]. With use of Equation (9) for two probability distributions $\mathbf{p}=\left(p_{1}, \ldots\right)$ and $\mathbf{P}=\left(P_{1} \ldots\right)$ we define the corresponding relative entropy as

$$
H(p \| P)=\sum_{i} p_{i}\left(-\ln \frac{P_{i}}{p_{i}}\right)^{q} .
$$

The proof that $H(p \| q)$ is positive follows along the same lines as the $q=1$ case. Provided that $\sum_{i} p_{i}=1$ and $f(x)$ is a convex function, the Jensen inequality

$$
\sum_{i} p_{i} f\left(x_{i}\right) \geq f\left(\sum_{i} p_{i} x_{i}\right),
$$

and the fact that $f(x)=(-\ln x)^{q}$ is a convex function allow us to write for Equation (12),

$$
\begin{aligned}
H(p \| P) & \geq\left(-\ln \sum_{i} p_{i} \frac{P_{i}}{p_{i}}\right)^{q} \\
& =\left(-\ln \sum_{i} P_{i}\right)^{q} \\
& =0 .
\end{aligned}
$$

Now, to extract the Fisher information measure for $q \neq 1$, we consider a distribution function $p(x)$ and a small shift $\Delta$ such that Equation (12) becomes

$$
H(p(x) \| p(x+\Delta))=\int d x p(x)\left(-\ln \frac{p(x+\Delta)}{p(x)}\right)^{q} .
$$

Expanding $\ln (p(x+\Delta)$ up to second order in $\Delta$,

$$
\begin{aligned}
H(p(x) \| p(x+\Delta)) & =-\int d x p(x)\left[\frac{p^{\prime}}{p} \Delta+\left(\frac{p^{\prime \prime}}{p}-\frac{p^{2}}{p^{2}}\right) \frac{\Delta^{2}}{2}\right]^{q} \\
& =-\int d x p(x) \Delta^{q} \sum_{n=0}\left(\begin{array}{l}
q \\
n
\end{array}\right)\left(\frac{p^{\prime}}{p}\right)^{q-n}\left[\left(\frac{p^{\prime \prime}}{p}-\frac{p^{2}}{p^{2}}\right) \frac{\Delta}{2}\right]^{n},
\end{aligned}
$$

where $p^{\prime}=\frac{d p}{d x}$. In order to obtain the Fisher information it is enough to keep the two lowest order terms,

$$
H(p(x) \| p(x+\Delta))=-\int d x\left(\left(\begin{array}{l}
q \\
0
\end{array}\right) \frac{p^{q}}{p^{q-1}} \Delta^{q}-\left(\begin{array}{l}
q \\
1
\end{array}\right)\left(\frac{p^{q+1}}{p^{q}}-\frac{p^{\prime \prime} p^{q-1}}{p^{q-1}}\right) \frac{\Delta^{q+1}}{2}\right),
$$

such that, up to a constant, we define

$$
I_{q}=\int d x \frac{p^{q+1}(x)}{p^{q}(x)} .
$$


In our case, after introducing the new function $\phi(x)=p^{\frac{1}{q+1}}(x)$, Equation (17) becomes

$$
I_{q}=(q+1)^{q+1} \int d x\left(\frac{d \phi}{d x}\right)^{\frac{q+1}{2}}\left(\frac{d \phi}{d x}\right)^{\frac{q+1}{2}},
$$

which reduces to Equation (4) when $q \rightarrow 1$. Setting $\delta I_{q}=0$ in Equation (18), leads to the equation

$$
\left(\frac{d \phi(x)}{d x}\right)^{q-1} \frac{d^{2} \phi(x)}{d x^{2}}=0 .
$$

Although solutions to Equation (19) are not different than the $q=1$ case, solutions to the corresponding eigenvalue problem are different. Let us consider the equation

$$
\left(\frac{d \phi(x)}{d x}\right)^{q-1} \frac{d^{2} \phi(x)}{d x^{2}}=\lambda \phi(x) .
$$

Solutions to Equation (20) are given by the function

$$
\phi(x)=A[1+(1-q) \gamma x]^{\omega},
$$

where $A, \gamma$ and $\omega$ are constants to be determined. Taking the elementary derivatives we find that $\omega=-\frac{q+1}{1-q}$ and $\gamma=\left(\frac{\lambda}{2(q+1)^{2 q-1}}\right)^{\frac{1}{2 q}}$, where solutions with $\gamma>0$ restrict the values of $q$ such that $(-1)^{\frac{q+1}{2 q}}=1$. It is interesting to remark that functions in Equation (21) are of the type of solutions found for the probability distribution in the non-extensive formulation of statistical mechanics [10] [11. The constant $A$ is found by normalizing the function in Equation (21) using the definition of the $\Gamma$ function, in a very similar way as done to calculate the partition function of non-extensive statistical mechanics [12 13,

$$
\Gamma(z)=x^{z} \int_{0}^{\infty} t^{z-1} e^{-t x} d t \quad x>0, z>0,
$$

leading to the value $A=\gamma(1+q)$.

Taking the limit $q \rightarrow 1$ the function in Equation (21) becomes the expected exponential solution $\phi(x ; q=1)=\lambda^{1 / 2} \exp \left(-\lambda^{1 / 2} x\right)$.

\section{Anomalous diffusion}

In the last twenty years, anomalous diffusion has been observed in a variety of interesting physical systems, for example breakable micelles dissolved in salted water [14, measurements of cardiac beat to beat intervals in healthy individuals 15], chaotic transport in a two-dimensional fluid flow in a rotating annulus 16], laser cooling of atoms to very low temperatures [17, and more recently several interesting experiments with living cells in which a transition from subdiffusive to superdiffusive behavior is observed [18 [19. On the other hand, several mathematical models have been proposed to study different aspects of 
anomalous diffusion. Some of these models are based on a linear differential equation for diffusion on fractals [20, linear and non-linear fractional differential equations [21]-27], a study of particle chaotic dynamics along a stochastic web [28, a study of super-diffusion in a hamiltonian system in the context of a continuous-time random walk approach to Lévy flights 29, nonlinear differential equations [30-35] and some calculations based on Tsallis non-extensive statistical mechanics [36]-40].

In our case, the simplest model we can propose is based on the differential equation

$$
K\left(\frac{\partial W}{\partial x}\right)^{q-1} \frac{\partial^{2} W}{\partial x^{2}}=\frac{\partial W}{\partial t}
$$

where $K$ is the diffusion constant. The solution to the eigenvalue problem in the previous section guides us to propose the function

$$
W(x, t)=\frac{A}{t^{\lambda}} \Psi(x, t)^{\omega}
$$

where

$$
\Psi(x, t)=\left[1+\frac{(1-q)}{C} \frac{x^{\gamma}}{t^{\beta}}\right],
$$

and $A, \lambda, C, \beta, \gamma$ and $\omega$ are constants to be determined, which in the $q \rightarrow 1$ limit must satisfy: $\lambda \rightarrow 1 / 2, \beta \rightarrow 1$ and $\gamma \rightarrow 2$. By performing the elementary derivatives, comparing powers in $\Psi(x, t), x$ and $t$ and the constants in both sides of Equation (23) we find that

$$
\begin{aligned}
\omega & =-\frac{q}{1-q} \\
\gamma & =\frac{q+1}{q} \\
\beta & =\frac{q+1}{2 q^{2}} \\
\lambda & =\frac{1}{2 q} \\
A^{1-q} & =\frac{2 K(q+1)^{q}}{C^{q}} .
\end{aligned}
$$

Similarly to the previous section there is a restriction on the values of $q$, due to the fact that in order to have the correct $q \rightarrow 1$ limit the constants $A$ and $C$ have to be positive leading to the set of allowed values of $q=\frac{2 n-1}{2 n+1}$ where $n=1,2,3 \ldots$. Since the solution $W(x, t)$ must have dimension $[W]=\frac{1}{\mathrm{~cm}}$, these constants have dimensions: $[K]=\frac{\mathrm{cm}^{2 q}}{\mathrm{~s}},[A]=\frac{\mathrm{s}^{\lambda}}{\mathrm{cm}}$ and $[C]=\frac{\mathrm{cm}^{\gamma}}{\mathrm{s}^{\beta}}$. In Fig. 1 we show the function $W(x, t)$ for the case of $q=5 / 7$ and $t=0.05,0.09$ and 0.2 , and in Fig. 2 the function $W(x, t=0.5)$ for the superdiffusive cases $q=5 / 7, q=19 / 21$ and the normal diffusive case $q=1$. 
As a next step we find an additional relation between the constants $A$ and $C$ by normalizing the solution $W(x, t)$

$$
\frac{A}{t^{\lambda}} \int_{-\infty}^{\infty} d x\left[1+\frac{(1-q)}{C} \frac{x^{\gamma}}{t^{\beta}}\right]^{\omega}=1
$$

Since $q$ takes values such that $(-1)^{\gamma}=1$ we can change the limits from $(-\infty, \infty)$ to $(0, \infty)$, such that with use of the integral representation of the $\Gamma$ function we obtain

$$
A=\left(\frac{1-q}{C}\right)^{1 / \gamma} g(q)
$$

where the function $g(q)=\frac{\gamma \Gamma(-\omega)}{2 \Gamma(1 / \gamma) \Gamma(-\omega-1 / \gamma)}$. Defining $Q=\frac{2 q^{2}}{1-q^{2}}$ and by using [4]

$$
\lim _{Q \rightarrow \infty} \frac{\Gamma\left(Q+\frac{1}{\gamma}\right)}{\Gamma(Q)} e^{-(1 / \gamma) \ln Q}=1,
$$

with Equation (30) we find that the constant $A$ has the correct normal diffusion limit $A \rightarrow \sqrt{\frac{1}{4 \pi K}}$ as $q \rightarrow 1$. The constant $C$ is given by

$$
C=(2 K)^{\lambda \gamma}(1+q)^{\gamma / 2}(1-q)^{1 / 2 \omega} g^{\gamma / 2 \omega}(q) .
$$

The mean squared displacement $<x^{2}>$ is obtained by solving

$$
<x^{2}>=\frac{A}{t^{\lambda}} \int_{-\infty}^{\infty} d x x^{2}\left[1+\frac{(1-q)}{C} \frac{x^{\gamma}}{t^{\beta}}\right]^{\omega},
$$

leading, up to a $q$-dependent constant, to

$$
<x^{2}>\sim K^{1 / q} t^{1 / q}, \quad 0<q<1 .
$$

Since $0<q<1$, our model seems to describe exclusively superdiffusive processes. This apparent drawback is due to the fact that we took in Equation (6) the particular value $n=1$. A general expression, $\forall n>0$ for the entropy function $S_{q}[p]$ reads

$$
S_{q}[p]=\lim _{t \rightarrow-1}\left(\frac{d}{d t}\right)^{n}\left(-\infty D_{t}^{q-n} \sum_{i} e^{-t \ln p_{i}}\right),
$$

which leads to the same mathematical function

$$
S_{q}[p]=\sum_{i} p_{i}\left(-\ln p_{i}\right)^{q},
$$

but now the parameter $q$ takes values in the set $n-1<q<n$. The solution for $q>1$ reads

$$
\begin{aligned}
W^{\prime}(x, t) & =\frac{A^{\prime}}{t^{\lambda}}\left[1-\frac{(q-1)}{C^{\prime}} \frac{x^{\gamma}}{t^{\beta}}\right]^{\omega} \\
& =0 \quad \text { for }\left(1-\frac{(q-1)}{C^{\prime}} \frac{x^{\gamma}}{t^{\beta}}\right)<0, \quad q>1,
\end{aligned}
$$


with the constants $A^{\prime}$ and $C^{\prime}$ given by

$$
\begin{aligned}
& A^{\prime}=\left[\frac{\gamma \lambda}{\omega^{q} K(\gamma-1)}\right]^{\lambda} g^{\prime}(q)^{\frac{\gamma}{2}} \\
& C^{\prime}=\left[\frac{(\gamma-1) K}{\lambda}\right]^{\frac{1}{q}-\frac{\lambda}{\omega}}(q-1) \omega^{\frac{\gamma}{2}} \gamma^{1+\frac{\lambda}{\omega}} g^{\prime}(q)^{\frac{\gamma}{2 \omega}},
\end{aligned}
$$

with $g^{\prime}(q)=\frac{\Gamma(1+\omega+1 / \gamma)}{2 \Gamma(1 / \gamma) \Gamma(1+\omega)}$. In order to normalize the new solution we used the integral representation of the $\Gamma$-function in the complex plane

$$
\frac{1}{\Gamma(z)}=\frac{i}{2 \pi} \oint_{C}(-t)^{-z} e^{-t} d t
$$

In this case, $q>1$, the constant $\omega$ is positive and therefore the solution $W^{\prime}(x, t)$ becomes the usual normal diffusion solution in the $q \rightarrow 1$ limit. The allowed values of $q$ are $q=\frac{2 n+1}{2 n-1}$, and thus Equation (36) for an arbitrary positive integer $n$, includes also the case of subdiffusive behavior. Fig. 3 displays the function $W^{\prime}(x, t=0.5)$ in Equation (39) for the subdiffusive cases $q=7 / 5, q=21 / 19$ and the normal diffusive case $q=1$.

It is interesting to remark that for the particular value $\gamma=2$ and arbitrary values for the constants $\omega, \lambda$ and $\beta$ the functions $W(x, t)$ and $W^{\prime}(x, t)$ in Equations (24) and (39) are of the same type of the solutions of the nonlinear diffusion equation studied in [30] 32] [42, called the Barenblatt-Pattle solution.

\section{Conclusions}

In this paper, based on a previously proposed entropy function $S_{q}[p]$ we first defined the corresponding relative entropy $H(p \| P)$, and by considering the two probability distributions to differ by a small global shift $\Delta$, we expanded the relative entropy with respect to this shift and extracted the Fisher information measure for $q \neq 1$. Then, following Frieden's idea we defined a new function $\phi(x)$ in terms of the probability $p(x)$ that allowed us to reinterpret the Fisher information as a free action $I_{q}$. Setting the variation of $I_{q}$ to $\delta I_{q}=0$ we find a differential operator that reduces to a second order derivative at $q=1$. We found that the eigenfunctions of this differential operator are of the same type than the probability distribution found in the Tsallis formulation of nonextensive statistical mechanics. Using this differential operator we proposed a simple differential equation that coincides with the normal diffusion equation in the $q \rightarrow 1$ limit. In our case, the solutions are such the exponent $\gamma$ in the $x$ variable is a fraction $\gamma=\frac{q+1}{q}$, taking values in the set $\gamma=\frac{4 n}{2 n-1}$ for $q=\frac{2 n-1}{2 n+1}$ (superdiffusion), and $\gamma=\frac{4 n}{2 n+1}$ for $q=\frac{2 n+1}{2 n-1}$ (subdiffusion), $\forall n=1,2,3 \ldots$ The work done in this paper is a first step to study anomalous diffusion with a rather simple and different mathematical model. In order to study anomalous diffusion in a more realistic way will require to introduce a time dependent source term (absorption) and external forces (drift) in a three dimensional version of this model, which we will attempt to address in future communications. 


\section{References}

[1] R. A. Fisher, Statistical Methods and Scientific Inference, Second Edition, (Oliver and Boyd, London, 1959).

[2] B. Roy Frieden, Physics from Fisher Information, (Cambridge University Press, 1998).

[3] B. Roy Frieden, B. H. Soffer, Phys. Rev. E 52 (1995) 2274.

[4] B. Roy Frieden, W. J. Cocke, Phys. Rev. E 54 (1996) 257.

[5] G. V. Vstovsky, Phys. Rev. E 51 (1995) 975.

[6] R. A. Fisher, Ann. Eugenics 12 (1943) 1.

[7] M. R. Ubriaco, Phys. Lett. A 373 (2009) 2516.

[8] S. Abe, Phys. Lett. A 24 (1997) 326.

[9] F. Jackson Quart. J. Pue Appl. Math 41 (1910) 193.

[10] C. Tsallis, J. Stat. Phys. 52 (1988)479.

[11] A list of works can be found in tsallis.cat.cbpf.br/biblio.htm.

[12] D. Prato, Phys. Lett. A 203 (1995) 165.

[13] S. Abe. Physica A 269 (1999) 403.

[14] A. Ott, J. P. Bouchaud, D. Langevin, W. Urbach, Phys. Rev. Lett. 65 (1990) 2201.

[15] C.-K. Peng, J. Mietus, J. M. Hausdorff, S. Havlin, H. E. Stanley, A. L. Golberger, Phys. Rev. Lett. 70 (1993) 1343.

[16] T. H. Solomon, E. R. Weeks, H. L. Swinney, Phys. Rev. Lett. 71 (1993) 3975 .

[17] F. Bardou, J. P. Bouchaud, O. Emile, A. Aspect, C. Cohen- Tannoudji, Phys. Rev. Lett. 72 (1994) 203.

[18] C. Raupach, D. P. Zitterbart, C. T. Mierke, C. Metzner, F. A. Müller, B. Fabry, Phys. Rev. E 76 (2007) 011918, and references therein.

[19] F. Gallet, D. Arcizet, P. Bohec, A. Richert, arXiv:bio-ph/0901.3087.

[20] B. O’Shaughnessy, I. Procaccia, Phys. Rev. Lett. 54 (1985) 455.

[21] W. R. Schneider, W. Wyss, J. Math. Phys. 30 (1989) 134.

[22] R. Meltzer, W. G. Glöcke, T. F. Nonnenmacher, Physica A 211 (1994) 13.

[23] M. Bologna, C. Tsallis, P. Grigolini, Phys. Rev. E 62 (2000) 2213. 
[24] E. K. Lenzi, L. C. Malacarne, R. S. Mendes, I. T. Pedron, Physica A 319 (2003) 245.

[25] A. J. Turski, B. Atamaniuk, E. Turska, arXiv:math-phys/0701068.

[26] A. V. Chechkin, V. Yu Gonchar, R. Gorenflo, N. Korabel, I. M. Sokolov, Phys. Rev. E 78 (2008) 021111.

[27] R. Metzler, J. Klafter, Phys. Rep. 339 (2000).

[28] G. M. Zaslavsky, D. Stevens, H. Weitzner, Phys . Rev. E 48 (1993) 1683.

[29] J. Klafter, G. Zumofen, Phys. Rev. E 49 (1994) 4873.

[30] J. G. Berryman, J. of Math. Phys. 18 (1977) 2108.

[31] J. Stephenson, Physica A 222 (1995) 234.

[32] A. Compte, D. Jou , J. Phys. A: Math. Gen. 29 (1996) 4321.

[33] L. C. Malacarne, R. S. Mendes, I. T. Pedron, E. K. Lenzi, Phys. Rev E 63 (2001) 030101(R).

[34] I. T. Pedron, R. S. Mendes, L. C. Malacarne, E. K. Lenzi, Phys. Rev. E 65 (2002) 041108.

[35] P. C. da Silva, L. R. da Silva, E. K. Lenzi, R. S. Mendes, L. C. Malacarne, Physica A 342 (2004) 16.

[36] A. R. Plastino, A. Plastino, Physica A 222 (1995) 347.

[37] C. Tsallis, D. J. Bukman, Phys. Rev. E 54 (1996) R2197.

[38] C. Essex, C. Schulzky, A. Franz, K. H. Hoffmann, Physica A 284 (2000) 299.

[39] E. Lutz, Phys. Rev. A 67 (2003) 051402(R).

[40] S. Abe, Phys. Rev. E 69 (2004) 016102.

[41] I. S. Gradshteyn, I. M. Ryzhik, Table of Integrals, Series and Products, (1965, Academic Press, New York) p. 937.

[42] J. L. Vázquez , The Porous Medium Equation: Mathematical Theory , Oxford Mathematical Monographs, (2007, Oxford, Clarendon). 
Fig. 1: The solution $W(x, t)$ to Equation (23) for the case of $q=5 / 7$ and $t=0.05,0.09$ and 0.2 .

Fig. 2: The function $W(x, t=0.5)$ for the superdiffusive cases $q=5 / 7$ (thin solid line), $q=19 / 21$ (dotted) and the normal diffusive case $q=1$.

Fig. 3: The function $W^{\prime}(x, t=0.5)$ in Equation (39) for the subdiffusive cases $q=7 / 5$ (thin solid line), $q=21 / 19$ (dotted line) and the normal diffusive case $q=1$. 

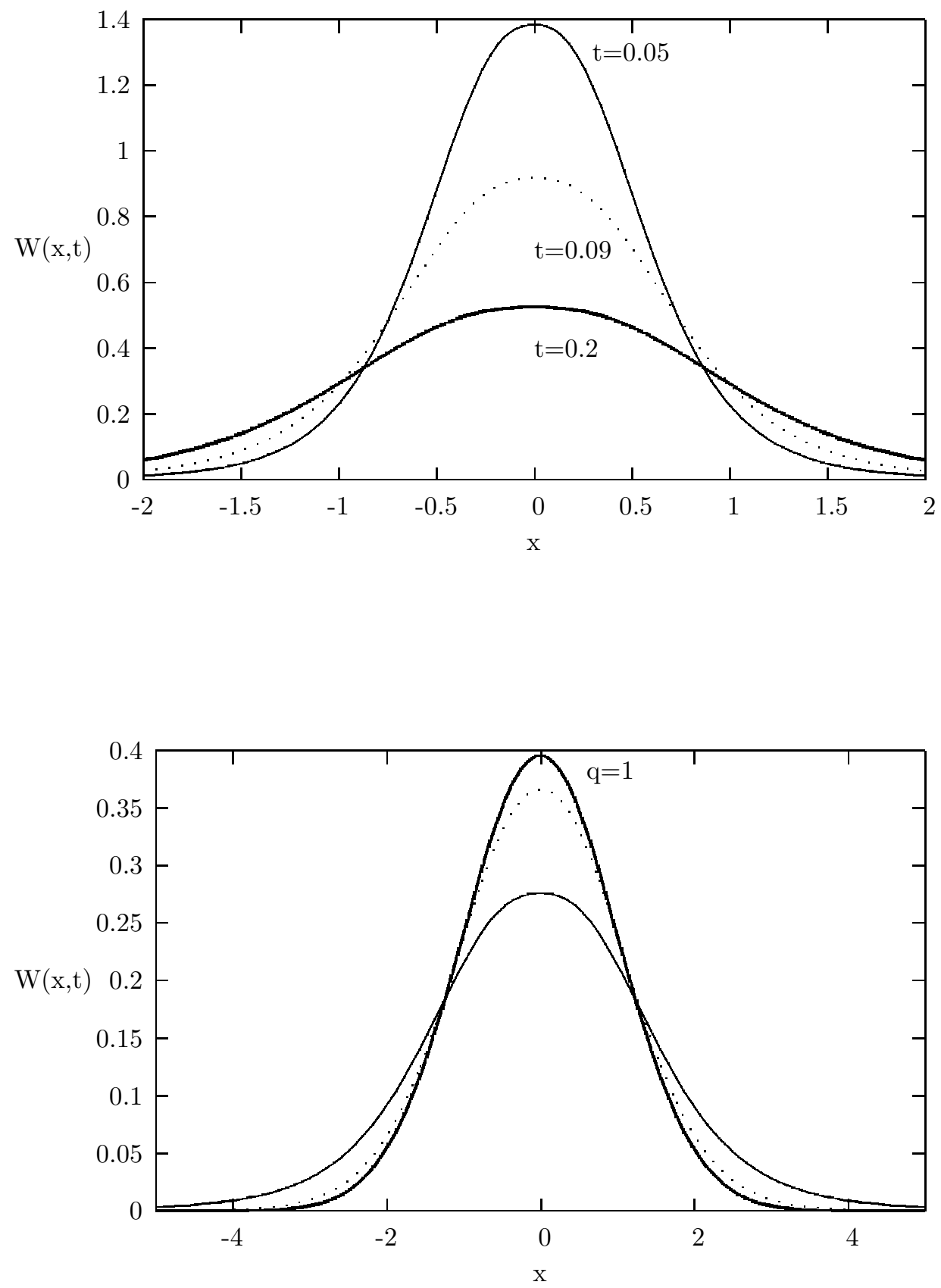


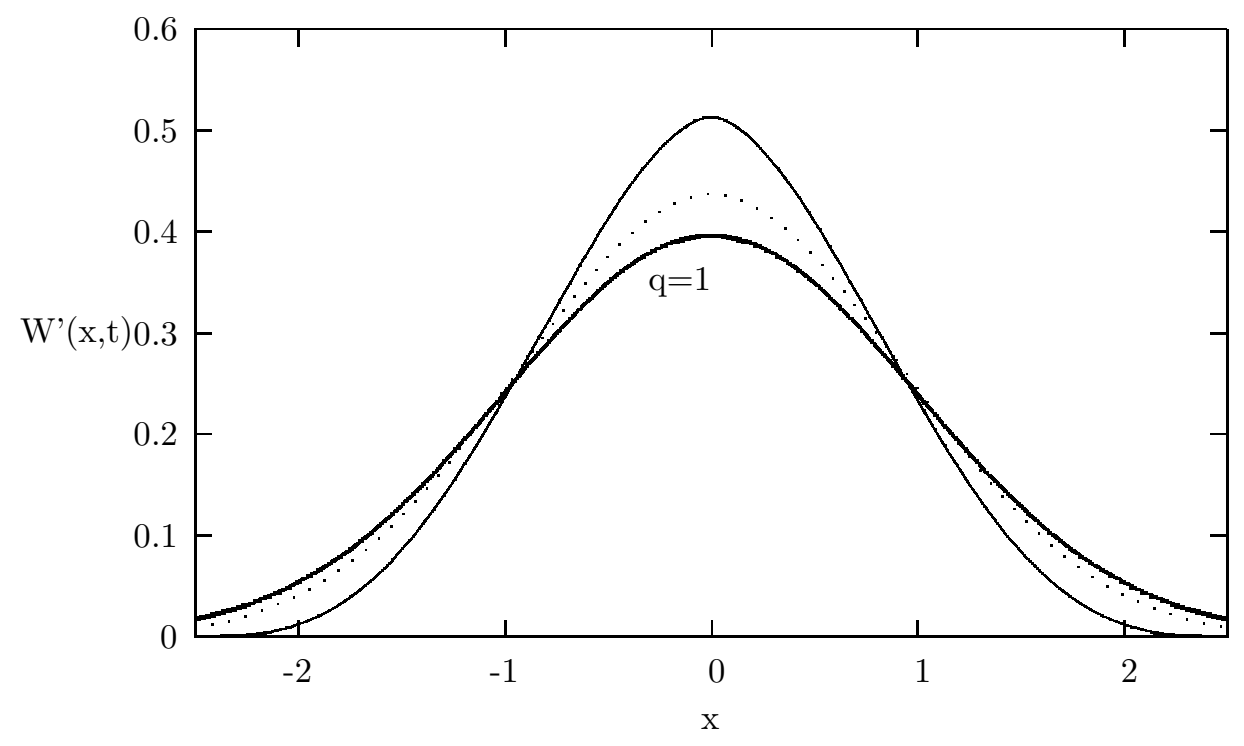

